\title{
Persistent Mullerian Duct Syndrome with Transverse Testicular Ectopia Presenting as an Irreducible Inguinal Hernia
}

\author{
Zoi Lamprinou*, Ioannis Skondras, Jonida Mene, Adelais Tzortzopoulou, Elisavet Kanna and Orthodoxos \\ Achilleos \\ $2^{\text {nd }}$ Department of Pediatric Surgery, P \& A Kyriakou Childrens' Hospital, Greece
}

Submission: December 30, 2021; Published: January 13, 2022

*Corresponding author: Zoi Lamprinou, 2nd Department of Pediatric Surgery, P \& A Kyriakou Childrens' Hospital, Thivon and Levadeias str., Goudi, 11527, Athens, Greece

\begin{abstract}
Jordan was the first who described persistent müllerian duct syndrome (PMDS) associated with transverse testicular ectopia (TTE) in 1895 [1]. The clinical presentation is a unilateral undescended testis from birth and an inguinal hernia on the contralateral hemiscrotum. We present the case of a newborn male, with history of bilateral cryptorchidism, presenting with a recent swelling in the left inguinal region. On examination the neonate had a left inguinal hernia and an empty right hemiscrotum. An inguinal approach revealed a hernia sac containing female-resembling gonads by intraabdominal approach. Hernia was repaired and biopsies taken from both gonads. A hormonological screening combined with histology results set the diagnosis of PMDS. Based on the current literature, we decided a second surgical intervention a year later so as to perform orchedopexy and müllerian remnants excision. A two-staged approach can prevent injuries of important structures such as vas deferens and resection of female gonads decreases the risk of malignancy.
\end{abstract}

Keywords: Transverse testicular ectopia; PMDS; Cryptorchidism

Abbreviations: PMDS: Persistent Müllerian Duct Syndrome; TTE: Transverse Testicular Ectopia; AMH: Anti-Müllerian Hormone; AMHR: AntiMüllerian Hormone Receptor

\section{Introduction}

PMDS is an uncommon disorder of sex differentiation in male fetuses. Testosterone and anti-Müllerian hormone (AMH) secreted by the testes during the 7 weeks of gestation are responsible for the prevalence of the Wolffian duct and the regression of Müllerian duct, respectively, in the male embryo.

Mutations of either AMH or AMH receptor's (AMHR) genes are the genetic basis of the syndrome which leads to persistence of Müllerian derivatives as uterus, fallopian tube, cervix and seldom the upper vagina. We present a rare case of a male newborn with a history of bilateral undescended testis which presented with left irreducibleinguinal hernia.

\section{Materials and Methods}

An 18 days old, full term male neonate with a history of bilateral impalpable testis, presented with swelling in the left groin area. The swelling was irreducible and extended in the left hemiscrotum. Symptoms of intestinal obstruction were not present. During ultrasound examination, both testes were present in the left hemiscrotum and an uterus-like structure was depicted in the deep inguinal ring. A diagnostic surgical intervention was decided directly (Figures $1 \& 2$ ).

A left inguinal transverse incision was performed and the hernia sac was identificated, edematous and fragile. Despite meticulous dissection the sac was teared and opened. The content of the sac was the two gonads (Figure 3) resembling ovaries accompanied by their fallopian tubes respectively and connected with the uterus. Content could not be reduced in the abdomen through the inguinal canal due to tissue edema. A Pfannenstiel incision was performed for a transabdominal approach. Contents of the hernia sac were set back in the abdomen and after getting biopsies from both gonads the internal inguinal ring was repaired. The patient discharged the fourth postoperative day without any complications. 


\section{Juniper Online Journal of Case Studies}

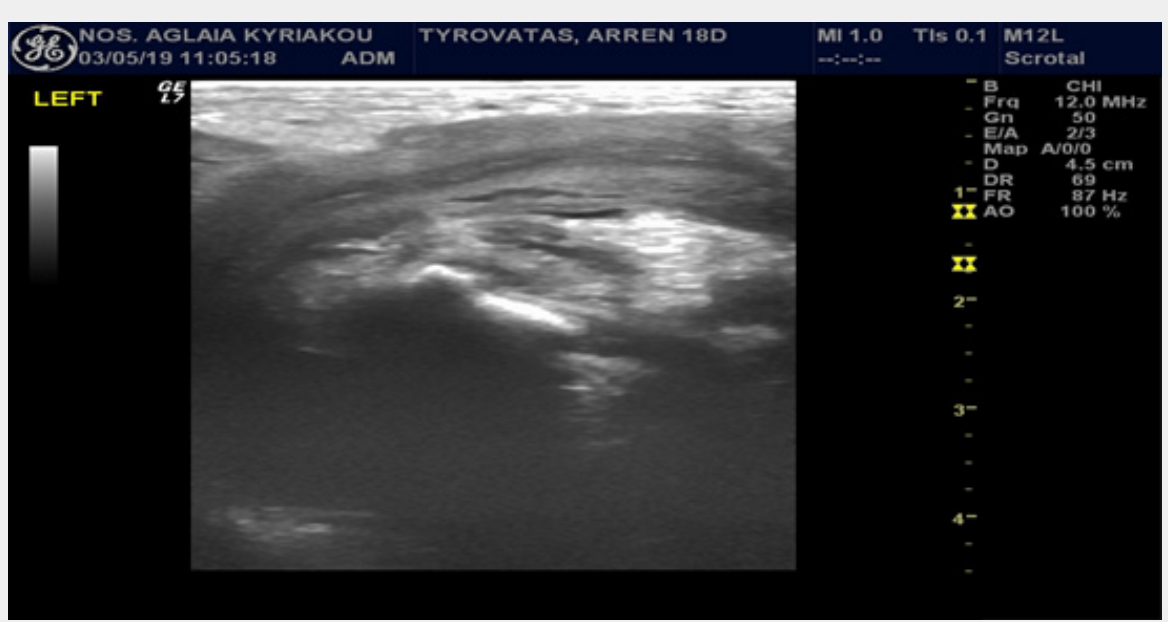

Figure 1: Solid structure in the left inguinal canal. Along this and in central position there is a linear hyperechogenic structure resembling endometrium.

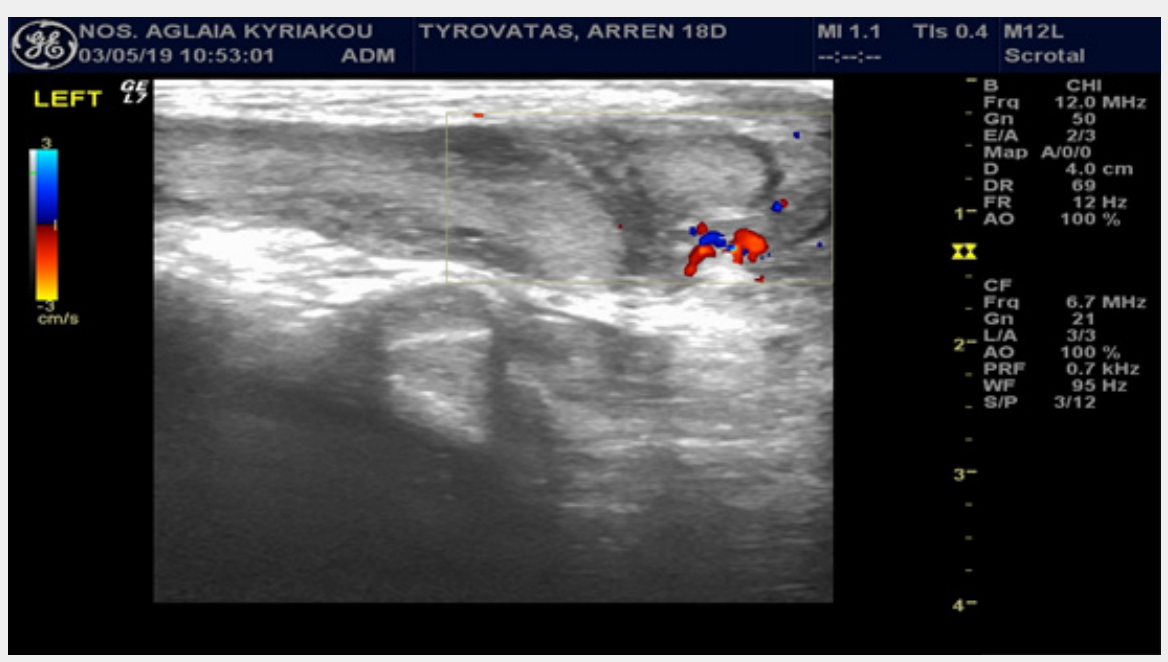

Figure 2: Both testicles in the left hemiscrotum with normal size and morphology.

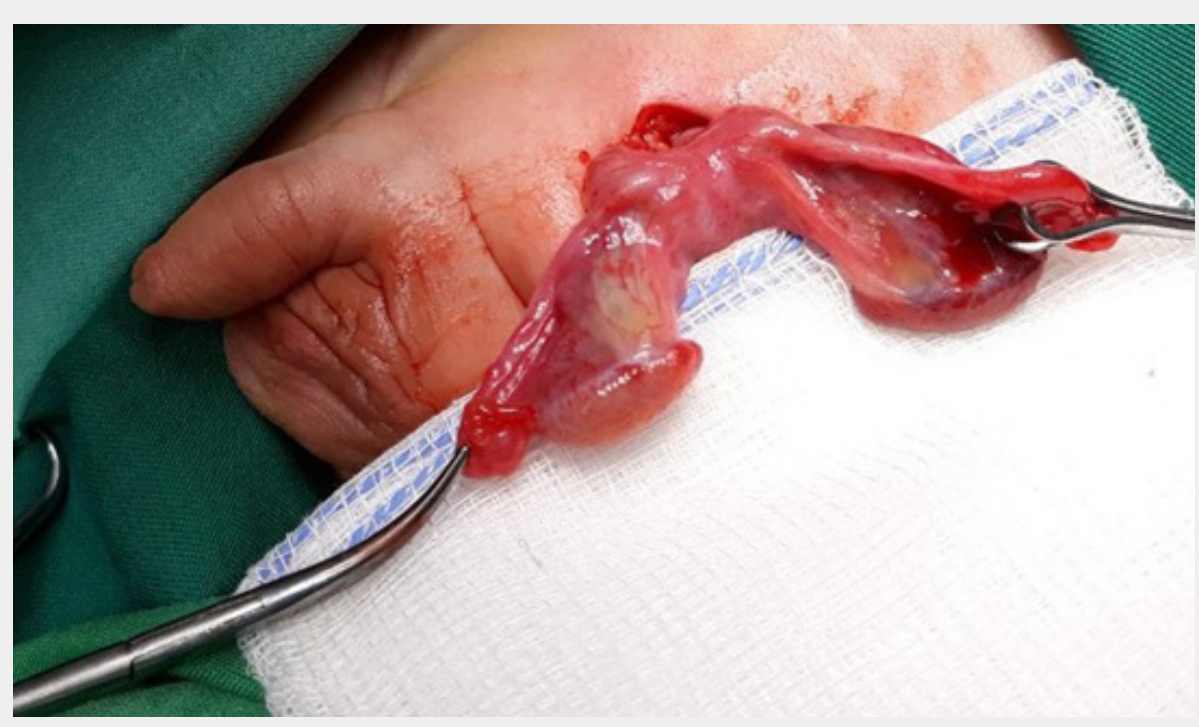

Figure 3: Contents of the hernia sac: gonads and Müllerian derivatives under inguinal open procedure. 
The histological examination results from the gonads' biopsies described them as immature testicular parenchyma. A surgical excision of the remnants was planned secondarily.

Postoperatively, a full hormonological work-up was carried out which revealed normal gonadotropins and testosterone levels, lack of estradiol and low levels of AMH. Patient had a normal karyotype $46 \mathrm{X}$, Y but genetic studies revealed that he was heterozygous for two recessive mutations on AMHR2 gene. The first one was a frequent deletion of 27 pairs of bases in the 10th of 11 exons of the gene, while the second one was a novel 2 base pair deletion mutation in the 6th exon.

At the age of 13 months old, the patient underwent surgical excision of the uterus, whose histological examination revealed a tubular fibromyous formation, whose lower part resembled vaginal and cervix epithelium and the upper part had similar morphology with endometrium. The gonads were mobilized and brought into the scrotum with preservation of both spermatic cords. A new ultrasound 3 months later depicted both testicles in the respective hemiscrotum.

\section{Discussion}

PMDS is a rare clinical entity of pseudohermaphrotidism, inherited in an autosomal recessive pattern. Male sex differentiation is driven by two hormones, each one secreted by different compartments of the fetal testis [2]. Fetal Leydig cells produce testosterone, which maintains Wolffian ducts and induces formation of the external male genitalia. AMH is synthesized by Sertoli cells and is responsible for the Müllerian duct regression. Mutations inactivating $\mathrm{AMH}$ or $\mathrm{AMH}$ receptor type 2(AMHR2) are responsible for PMDS [3]. Patients have a $46 \mathrm{XY}$ karyotype and due to normal testosterone secretion, their external genitalia are normally virilized. Nevertheless, Globa et al. [4] describe the unique case of two siblings with PMDS related to AMHR2 pathogenic variants and hypospadias. This finding should be investigated more as hypospadias could be a part of the clinical manifestations of the syndrome.

The syndrome can be classified in 3 types based on the anatomical position of the testes and the Müllerian remnants [2,5] (Table 1). Our case is included in the third type and is known as transverse testicular ectopia. In this anomaly, both testes have herniated in a common sac, in the same hemiscrotum.

Table 1: PMDS classification

\begin{tabular}{|c|c|c|}
\hline & Frequency & Clinical Presentation \\
\hline Type 1 (Male type) & $80-90 \%$ & $\begin{array}{c}\text { Unilateral cryptorchidism. The other testis is contained in a hernia sac with the uterus and one } \\
\text { fallopian tube. }\end{array}$ \\
\hline Type 2 (Female type) & $10-20 \%$ & Bilateral cryptorchidism. Both testes in an "ovarian-like" position in the pelvis. \\
\hline Type 3 (TTE) & $<5 \%$ & Both testes herniated in a common sac in the same hemiscrotum. \\
\hline
\end{tabular}

Ultrasonogrophy is the imaging modality of choice and sometimes can set the suspicion of the syndrome but subsequent MRI can also be helpful [6]. In the majority of the cases the correct diagnosis cannot be made preoperatively and explorative surgical intervention is inevitable.

In all cases, a full endocrinology investigation should be carried out either when the syndrome is suspected or after surgical intervention. As previously mentioned, it is known that PMDS is the result of mutations inactivating AMH or its receptor AMHR2. This does not affect Leydig cell function and testosterone production. Consecutively, patients with the syndrome have low AMH serum concentration but normal testosterone levels [7]. Notice should be given to postpubertal and, seldom, newborn males because they may have physiologically low AMH levels [2].

The main treatment considerations that should be taken into account is fertility and the risk of malignancy $[3,5]$.

Infertility is the most common complication of PMDS, although Picard et al. [2] mention that the percentage of fertility in patients range from $11 \%$ to $19 \%$. There is a single report in literature by Prakash et al. [8], describing a patient with PMDS with normal fertility before orchidopexy. Possible causes of infertility are 1) long-term cryptorchidism, 2) gonads' structural abnormalities and 3) vas deferens injury during surgical repair. Malignant degeneration of both testicles and Müllerian derivatives is a potential risk that should not be ignored from the surgeon.

As it regards the surgical management of the patient there are two issues that emerge from the above. The first issue is what kind of surgical intervention is appropriate when the syndrome is suspected. Usually, the Müllerian remnants are discovered incidentally during hernia repair or correction of cryptorchidism. Our recommendation, based in the recent literature, is that the surgical approach should be 2-staged [4,9-11] but there are authors as Telli et al. [12] who suggest one-stage treatment. The initial operation, which can be performed laparoscopically, when possible, should include biopsies taken from the gonads and the Müllerian remnants and hernia repair. The next stage includes orchidopexy and, arguably, dissection of the Müllerian structures. The second concern is if excision of Müllerian derivatives is necessary. In the past, excision was not mandatory as the surgical maneuvers could put at stake the vasa deferentia and the deferential blood supply of the testis [13]. Nowadays, due to 
increasing cases of malignancy arising from the female gonads, surgical removal of the remnants is the common practice that can be accomplished either laparoscopically or, if it is possible, with a robotic assisted approach [14]. Laparoscopic orchidopexy and excision is the preferable approach in the majority of pediatric surgery centers [11], and although we chose an open approach, we recommend laparoscopy as the method of choice. Different laparoscopic procedures can be used as the optimal result is orchidopexy with an intact vas of adequate length [15].

Long-term follow-up is necessary because malignant degeneration usually occurs after puberty, especially if the Müllerian derivatives haven't been removed. Patients with intact Müllerian duct or impalpable testes should undergo CT, MRI or endorectal ultrasound study even during adult life [15].

\section{Conclusion}

PMDS is a rare clinical entity, although, it should always be suspected in patients with nonpalpable undescended testis and contralateral inguinal hernia or in patients with bilateral cryptorchidism. The surgeon dealing with such case should bear in mind that two-stage surgical intervention is a safer option as simultaneous herniorraphy with orchidopexy and Müllerian structures excision can lead to spermatic vessels or vas deferens injury. Due to increased risk of malignancy, Müllerian remnants' removal is suggested in all cases. Open intraabdominal approach is a safe method of surgical excision in all cases, but in experienced centers, laparoscopic procedure can be used as the method of choice.

\section{References}

1. Jordan M (1895) Ein Fall von einseitigem Descensus testiculorum (Ectopia testis transversa). Dtsch Med Wochenschr 21(33): 525-526.

2. Picard JY, Cate RL, Racine C, Josso N (2017) The Persistent Müllerian Duct Syndrome: An Update Based Upon a Personal Experience of 157 Cases. Sex Dev 11(3): 109-125.
3. Picard JY, Josso N (2019) Persistent Müllerian duct syndrome: an update. Reprod Fertil Dev 31(7): 1240-1245.

4. Globa E, Zelinska N, Siryk N, Bashamboo A, McElreavey K (2020) Atypical Clinical Presentation of Persistent Müllerian Duct Syndrome in Siblings. Sex Dev 14(1-6): 27-32.

5. Saleem M, Ather U, Mirza B, Iqbal S, Sheikh A, et al. (2016) Persistent mullerian duct syndrome: A 24-year experience. J Pediatr Surg 51(10): 1721-1724.

6. Gutte AA, Pendharkar PS, Sorte SZ (2008) Transverse testicular ectopia associated with persistent Mullerian duct syndrome - the role of imaging. Br J Radial 81(967): e176-e178.

7. Xu HY, Zhang HX, Xiao Z, Qiao J, Li R (2019) Regulation of anti-Müllerian hormone (AMH) in males and the associations of serum AMH with the disorders of male fertility. Asian J Androl 21(2): 109-114.

8. Prakash N, Khurana A, Narula B (2009) Persistent Müllerian duct syndrome. Indian J Pathol Microbiol 52(4): 546-548.

9. Meyer T, Adam P, Höcht B (2010) Persistent Mullerian duct syndrome: a case-based algorithm. Eur J Pediatr Surg 20(3): 194-195.

10. Gupta A, Panda N, Saha ML, Ganguly S, Bandyopadhyay, et al. (2013) Persistent Mullerian duct syndrome with transverse testicular ectopia: rare presentation of inguinal hernia. Urol J 10(2): 909-911.

11. Farikullah J, Ehtisham S, Nappo S, Patel L, Hennayake S (2012) Persistent Müllerian duct syndrome: lessons learned from managing a series of eight patients over a 10-year period and review of literature regarding malignant risk from the Müllerian remnants. BJU Int 110(11 Pt C): E1084-E1089.

12. Telli O, Gökçe MI, Haciyev P, Soygür T, Burgu B (2014) Transverse testicular ectopia: a rare presentation with persistent Müllerian duct syndrome. J Clin Res Pediatr Endocrinol 6(3): 180-182.

13. Wuerstle M, Lesser T, Hurwitz R, Applebaum H, Lee SL (2007) Persistent mullerian duct syndrome and transverse testicular ectopia: embryology, presentation, and management. J Pediatr Surg 42(12): 2116-2119.

14. Lima M, Maffi M, Di Salvo N, Ruggeri G, Libri M, et al. (2018) Robotic removal of Müllerian duct remnants in pediatric patients: our experience and a review of the literature. Pediatr Med Chir 40(1).

15. Parelkar SV, Gupta RK, Oak S, Sanghvi B, Kaltari D, et al. (2009) Laparoscopic management of persistent mullerian duct syndrome. J Pediatr Surg 44(9): e1-e3.

\section{Your next submission with Juniper Publishers} will reach you the below assets

- Quality Editorial service

- Swift Peer Review

- Reprints availability

- E-prints Service

- Manuscript Podcast for convenient understanding

- Global attainment for your research

- Manuscript accessibility in different formats ( Pdf, E-pub, Full Text, Audio)

- Unceasing customer service

Track the below URL for one-step submission https://juniperpublishers.com/online-submission.php 